\title{
Glaucoma as Neurodegeneration in the Brain
}

This article was published in the following Dove Press journal:

Eye and Brain

Jane W Chan (D)

Noel CY Chan ${ }^{2}$

Alfredo A Sadun ${ }^{1,3}$

'Department of Ophthalmology, Doheny Eye Institute, Pasadena, CA, USA:

${ }^{2}$ Department of Ophthalmology \& Visual Sciences, The Chinese University of Hong Kong, Prince of Wales Hospital, Shatin, N.T., Hong Kong, People's Republic of China; ${ }^{3}$ Department of Ophthalmology, University of California, Los Angeles, CA, USA
Correspondence: Jane W Chan

Tel +I 7022909550

$\mathrm{Fax}+\mathrm{I} 6268174745$

Email janechan098@gmail.com

\begin{abstract}
Glaucoma, a group of diseases characterized by progressive optic nerve degeneration that results in irreversible blindness, can be considered a neurodegenerative disorder of both the eye and the brain. Increasing evidence from human and animal studies have shown that glaucoma shares some common neurodegenerative pathways with Alzheimer's disease (AD) and other tauopathies, such as chronic traumatic encephalopathy (CTE) and frontotemporal dementia. This hypothesis is based on the focal adhesion pathway hypothesis and the spreading hypothesis of tau. Not only has the Apolipoprotein E (APOE) gene been shown to be associated with $\mathrm{AD}$, but also with primary open angle glaucoma (POAG). This review will highlight the relevant literature in the past 20 years from PubMed that show the pathogenic overlap between POAG and AD. Neurodegenerative pathways that contribute to transsynaptic neurodegeneration in $\mathrm{AD}$ and other tauopathies might also be similar to those in glaucomatous neurodegeneration.
\end{abstract}

Keywords: primary open-angle glaucoma, tauopathy, amyloid precursor protein, phosphorylated tau, Alzheimer's disease

\section{Introduction}

Intraocular pressure (IOP) is the only known modifiable major risk factor for glaucoma, yet progression of visual loss remains inevitable in a subgroup of these patients even when conventional medical and surgical therapies optimize the IOP. The non-IOP factors contributing to visual loss include neuroinflammation, oxidative stress, dysregulation of calcium-dependent processes, defective autophagy, reactive gliosis, translaminar cribrosa pressure differences, and possibly the spreading of misfolded proteins. ${ }^{1}$ Some neurodegenerative pathways in primary open angle glaucoma (POAG) appear to overlap with those in Alzheimer's disease (AD) and other tauopathies, such as chronic traumatic encephalopathy (CTE). Synaptic dysfunction and synaptic remodeling have recently been implicated as key pathogenic mechanisms in Alzheimer's disease (AD). ${ }^{2}$ Two prevailing possible explanations for the synaptic dysfunction in $\mathrm{AD}$ and other tauopathies are the focal adhesion circular pathway hypothesis and the spreading hypothesis of tau.

Several genes involved in regulating the metabolic pathways of amyloid precursor protein (APP) and amyloid-beta (A $\beta$ ) also control the post-translational modification of tau protein species. ${ }^{2,3}$ Some AD genes, such as Apolipoprotein E (APOE), have been shown to increase the risk for POAG. ${ }^{4,5}$ Similar to the chronic insult of repetitive head trauma in chronic traumatic encephalopathy (CTE), ${ }^{3}$ recurrent IOP elevations might cause repetitive compressive compromise to the optic nerve head. These repetitive forces to the optic nerve head might potentially lead to tau accumulation, altered phosphorylation, and mis-sorting in glaucomatous neurodegeneration. Since 
transsynaptic neurodegeneration has been hypothesized to occur through the spreading of tau in AD, it might also explain the common neuropathologic involvement of the eye and brain in glaucomatous neurodegeneration.

\section{The Focal Adhesion Circular Pathway Hypothesis in Synaptic Dysfunction}

The classic amyloid cascade hypothesis proposed that defective APP metabolism leads to increased $A \beta$ production, in which $A \beta$ oligomers incite the spreading of tau from one neuron to another to cause synaptic and neuritic dysfunction. This linear model of the amyloid cascade hypothesis has recently taken a back seat to a vicious circle where dysfunctional metabolic pathways involving APP, tau, A $\beta$, and synaptic regulation revolve around the "focal adhesion core" (Figure 1). ${ }^{2,5}$ The focal adhesion pathway modulates the filamentous actin network by regulating actin-binding proteins, such as cofilin, that control dendritic spine morphology. This pathway depends on integrin to remodel the dendritic spine morphology for synaptic plasticity. ${ }^{2}$ This circular model takes into account the findings from genome-wide association studies, that genetic risk factors influence up to $80 \%$ of attributable risk in the more common forms of $\mathrm{AD}^{6}$ The dysregulation of synaptic function and downstream cellular signaling through the focal adhesion circular pathway may be an important part of the pathogenesis of $A D$. The primary dysfunction in $\mathrm{AD}$ is located at the synapse where increased oligomeric amyloid-beta peptide causes $N$-methyl-

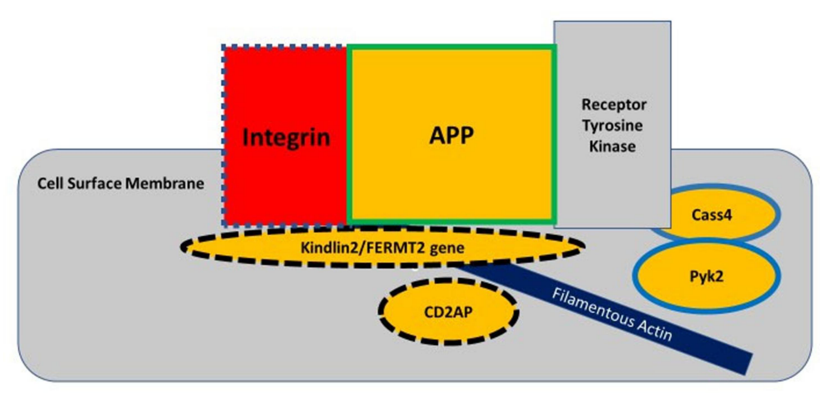

Figure I The focal adhesion complex regulates the actin cytoskeleton and downstream cell signaling pathways, such as in the spread of tau pathology. Integrin, amyloid precursor protein (APP), and receptor tyrosine kinase interact at the cell surface membrane to modulate cell adhesion. The genes for integrin, kindlin2, and CD2AP proteins regulate both APP metabolism and tau metabolism, as indicated by dashed outlines; whereas the genes for Cass 4 and Pyk2 proteins are involved in only taurelated pathways and the gene for APP only in APP-related pathways. The genes for proteins inside the yellow-colored shapes are considered risk factors for AD. Adapted from Dourlen P, Kilinc D, Malmanche Net al The new genetic landscape of Alzheimer's disease: from amyloid cascade to genetically driven synaptic failure hypothesis? Acta Neuropathol 138, 22I-236 (2019). Creative Commons Attribution 4.0 International License (http://creativecommons.org/licenses/by/4.0/). ${ }^{2}$
D-aspartate (NMDA) receptor-dependent synaptic depression and loss of dendritic spines. Cell-surface APP is required for the regulation of normal synaptic function and is located in the focal adhesion complex where it recruits various intracellular proteins to modulate integrin signaling. ${ }^{7}$ APP interacts with integrin for the development of neurite outgrowth and serves as a synaptic adhesion molecule at the pre- and post-synaptic membranes for the proper connection with other synapses; and for the development of normal synaptic morphology and spine density. ${ }^{7}$ Therefore, cell-surface APP located in the focal adhesion complex serves a physiologic function in maintaining normal synaptic function, whereas the accumulated oligomeric amyloid-beta peptides in this complex can lead to synaptic loss.

Any of the AD genes regulating APP metabolism, such as FERMT2, Cass4, CD2AP, and the ones regulating the actin cytoskeleton network, such as Kindlin-2, PUk2, CD2AP, and BIN1, can disrupt APP function and/or its interaction along the focal adhesion pathway. ${ }^{2}$ Amyloid-beta fibrils have been shown to activate integrin signaling to increase N-methyld-aspartate receptor (NMDAR) sensitivity to ultimately mediate $\mathrm{A} ß$ - induced neurotoxicity in hippocampal neurons. ${ }^{8}$ The scaffolding protein Ran BP9, that dephosphorylates cofilin, is a key regulator of actin filament dynamics, and it has been shown to lead to the formation of cofilin-actin rods in distal dendrites of synapses. ${ }^{9,10}$ Ran BP9 simultaneously increases APP and $B 1$-integrin endocytosis to cause elevated Aß production that then disrupts the focal adhesion pathway. ${ }^{11}$ This evidence supports the notion that cell surface APP plays an important role in synaptic function. Although microglia have been shown to form a protective barrier around amyloid deposits by compacting the fibrils into a less toxic form, ${ }^{12}$ microglial dysfunction can also influence the focal adhesion pathway through disrupted $A \beta$ clearance, leading to increased toxicity in synapses and early synaptic loss/remodeling. The focal adhesion pathway does not preclude the spreading hypothesis, which might involve a downstream trigger of tau-related excitotoxicity causing synaptic failure. Because the focal adhesion pathway is reiterative, it appears to recapitulate the vicious cycle of neurodegeneration involving the dysregulation of APP and tau.

\section{The Spreading Hypothesis of Tau in Synaptic Dysfunction}

Tau is the major microtubule-associated protein expressed in neurons and retinal ganglion cells (RGCs) that is regulated by the microtubule-associated protein tau (MAPT) gene on the long arm of chromosome 17q21; it has 16 
exons. $^{13}$ Post-translational alternative splicing of its mRNA produces six tau isoforms in the adult human brain. ${ }^{13-15}$ The normal physiological function of tau is to promote the assembly of tubulin to form microtubules and to help stabilize microtubule polymers involved in intracellular axonal transport. ${ }^{16,17}$ The spreading hypothesis of tau in $\mathrm{AD}$ proposes that tau undergoes post-translational modifications, such as hyperphosphorylation, which is catalyzed by Pyk2 (tyrosine kinase), one of the proteins in the focal adhesion complex, ${ }^{2}$ to cause its detachment from microtubules that then disassemble. Within the focal adhesion circular pathway, it is postulated that APP interacts with phosphorylated tau ( $\mathrm{p}$-tau) to initiate the process of taumisfolding and aggregation that then leads to increased NMDAR sensitivity, followed by dendritic spine degradation. $^{2}$ The p-tau is released from the dendrites into the extracellular space. ${ }^{18}$ The exact mechanism of abnormal tau propagation amongst neurons in the CNS is still not well understood at this time. ${ }^{19}$ Whether tau is spread from one neuron to another in POAG or in $\mathrm{AD}$ remains unproven.

\section{The Role of Apolipoprotein E (APOE) Gene in Primary Open-Angle Glaucoma (POAG)}

Genome-wide association studies (GWAS) have shown that the APOE gene $\varepsilon 2 / \varepsilon 3 / \varepsilon 4$ polymorphism has been associated with the focal adhesion pathway in AD. Although not all the AD genes participating in the focal adhesion pathway model have been shown to increase the risk of POAG, the APOE $\varepsilon 4 \varepsilon 4$ has been significantly associated with POAG. In a meta-analysis of fifteen studies including 2700 cases and 2365 controls from the literature since January 2014, the APOE $\varepsilon 4 \varepsilon 4$ genotype was found to be significantly associated with an increased risk of POAG in Asians, but not in Caucasians ( $\mathrm{P}=$ 0.003). ${ }^{5}$ Apolipoprotein E plays a key role in lipid metabolism, cholesterol transport, protein synthesis, tissue repair, cell growth and differentiation, and immune regulation. ${ }^{20-22}$ Apolipoprotein $\mathrm{E}$ binds to the lowdensity lipoprotein receptor to regulate the clearance of extracellular $A \beta$ across the blood-brain barrier and the blood-ocular barrier. An increased $A \beta$ deposition in the cerebral blood vessels and plaques has been shown on histopathology in AD brains with the APOE $\varepsilon 4 \varepsilon 4$ genotype. ${ }^{23}$ A more recent study showed the opposite finding, such that APOE $\varepsilon 4$ decreased the risk of POAG, while it increased the risk of AD. These conflicting findings regarding APOE $\varepsilon 4$ as a risk factor for POAG were thought to be attributed to the over-representation of normal tension glaucoma (NTG) in previous studies with mainly Asian subjects. ${ }^{24}$

Based on another meta-analysis of 108 case control studies with 35,389 POAG patients and 51,742 controls, the APOE gene was one of the 12 genes associated with an elevated risk of POAG, ${ }^{25}$ particularly APOE (-219G) with more optic nerve damage. APOE $(-491 \mathrm{~T})$ also interacts with the myocilin (MYOC) promoter $(-1000 \mathrm{G})$ which is associated with an increased risk of ocular hypertension in POAG. Based on the pooled data, the rs449647 of the APOE gene had a significant association with POAG in the allelic, homozygote, heterozygote, and dominant comparisons. In a more recent GWAS study on 2320 patients of African ancestry with POAG and on 2121 without $\mathrm{POAG}^{4}$ the $\mathrm{A} \beta \mathrm{A} 4$ precursor protein-binding family B member 2 (APBB2), located on chromosome 4 with the variant $\mathrm{rs} 59892895 \mathrm{~T}>\mathrm{C}$, was significantly associated with POAG $\left(\mathrm{P}=2 \times 10^{-8}\right)$. Each copy of the rs $59892895^{*} \mathrm{C}$ risk allele, most frequently found in individuals of African ancestry compared to those of European or Asian ancestry, was associated with an increased risk of POAG. The APBB2 gene is one of the genes regulating the processing of APP. Increased APBB2 gene expression was related to increased $\beta$-amyloid plaque deposition in POAG human retinal and primary visual cortex tissues. Therefore, defective clearance of $A B$ in the optic nerve might contribute to the development of POAG.

\section{Evidence for Abnormal Metabolism of Amyloid-Beta $(A B)$ and Tau in Glaucomatous Neurodegeneration}

Recent epidemiologic studies have reported a potential association between glaucoma and $\mathrm{AD} .{ }^{26-29}$ These studies support that glaucoma and $\mathrm{AD}$ might share similar pathophysiologic pathways in the metabolism of $A B$ and tau protein species. The histopathological defining features of $\mathrm{AD}$ are extracellular $\mathrm{A} \beta$ plaques and intracellular neurofibrillary tangles consisting of phosphorylated tau (ptau).$^{30}$ In glaucoma animal models, $A ß$ has been shown to play a role in RGC apoptosis. ${ }^{31}$ Not only have ptau deposits been demonstrated in human glaucomatous retinas, ${ }^{32}$ but further evidence of $A B$ deposits in the retina of POAG patients have also been shown to be consistent with decreased vitreous $A ß$ levels. ${ }^{33}$ 
Abnormal tau deposition in the retina has been found in 11 enucleated human eyes with a history of uncontrolled primary and secondary open-angle glaucoma compared to 10 age-matched controls. ${ }^{32}$ The immunofluorescent intensity for hyperphosphorylated tau, in the form of AT8 (phosphor-epitope at serine 202) was significantly higher in the outer region of the inner nuclear layer compared to controls. The optic nerve heads, however, did not show evidence of AT8 immunoreactivity. Normal tau immunoreactivity was absent in the glaucomatous retina, whereas it was present in all the control eyes. Normal tau was seen in the inner nuclear, inner plexiform layers, and less in RGCs and the retinal nerve fiber layer. ${ }^{32}$

Further evidence of abnormal beta-amyloid and abnormal tau accumulation in eyes have been demonstrated in animal models of ocular hypertension. In a rhesus monkey model of glaucoma, ${ }^{34}$ both eyes were induced to have elevated intraocular pressure by laser photocoagulation, and extracellular amyloid-beta ${ }_{1-42}$ and intracellular abnormal phosphorylated tau (AT8) deposits were observed in the lateral genicular nucleus (LGN). More neurofibrillary tangles and amyloid plaques, like those in $\mathrm{AD}$, were seen in the LGN than in the primary visual cortex, V1, which could imply that the disease progresses transsynaptically from the anterior to posterior visual pathways. ${ }^{34}$ In another study by Chiasseu, M. et al, tau accumulation, altered phosphorylation, and tau mis-sorting were shown in a rat glaucoma model. ${ }^{35}$ Like AD, glaucoma, and other tauopathies that progress with advancing age, tau accumulation in the rat retina increased with age and ocular hypertension, ${ }^{36,37}$ as compared with age-matched controls. It is known that different types of oxidative stress can lead to hyper-, hypo-, or de-phosphorylation of tau species. ${ }^{38}$ As shown in $\mathrm{AD}$ and other tauopathies, the ocular hypertension rat models demonstrate that phosphorylation of tau residues S396 and S404 is increased, but S199 phosphorylation is decreased, relative to the total tau levels in the retina. ${ }^{3}$ Chiasseu et $\mathrm{al}^{33}$ also showed that tau monomers likely converted to oligomers in the glaucomatous retinas because there was no evidence of transcriptional upregulation by increased mRNAs for these oligomers. Without any increase in retinal tau mRNA, they further hypothesized that tau accumulation in non-RGCs could be due to impaired autophagy or proteasomal degradation and/or spreading from one cell to another. Tau oligomers, rather than tau monomers and neurofibrillary tangles, were implicated as the main toxic forms that initiate and propagate glaucomatous degeneration..$^{33,39,40}$ Like $\mathrm{AD}$ and other tauopathies, considerable tau mis-sorting was also observed in these glaucomatous rat retinas. The abnormal forms of tau were found in RGC dendrites and not in axons, suggesting that the abnormal tau was mis-sorted from axons to dendrites in the glaucomatous rat retinas, instead of anterogradely from RGC somas to the axons. ${ }^{41}$ It has also been hypothesized that impaired anterograde axonal transport could lead to tau accumulation in dendrites, where it could interact with postsynaptic protein Fyn and with A-beta to mediate excitotoxicity in the microtubules. ${ }^{42}$ These proposed neurodegenerative pathways are consistent with a previous study showing that elevated IOP can initiate damage to RGC dendrites via tau mis-sorting. ${ }^{43}$ Other studies also support the idea that remodeling of the RGC dendritic circuitry and axonal loss and synaptic dysfunction precedes RGC death in glaucoma. ${ }^{44,45}$ It, therefore, appears that tau dysregulation in these experimental glaucomatous models might recapitulate some of the key common pathological features in human glaucoma, $\mathrm{AD}$, and other tauopathies.

\section{Retinotopic Transsynaptic Neurodegeneration in Glaucoma}

Elevated IOP is a major risk factor for the progression of glaucoma that leads to impaired axonal transport and synaptic degenerative changes that results in RGC apoptosis. ${ }^{46,47}$ In a mouse model of ocular hypertension, anterograde axonal transport deficits were observed to precede retrograde deficits located at the optic nerve head. ${ }^{48}$ Experimental glaucoma models have also demonstrated a significant correlation between mean IOP and the degree of LGN neuronal loss, ${ }^{49}$ and a higher mean IOP duration reduced the time to neuronal loss. ${ }^{50}$ CaMKII- $\alpha$ isoform (calcium/calmodulin-dependent kinase type IIalpha), an important post-synaptic density protein that regulates synaptic strength and the density of glutaminergic synapses, and its absence can lead to synaptic dysfunction and synaptic loss. ${ }^{51,52}$ Elevated IOP with or without optic nerve fiber loss was shown to reduce the expression of this protein in koniocellular neurons,${ }^{49}$ leading to the theory of impaired transsynaptic changes in LGN in early glaucoma.

It has been demonstrated that atrophy of the posterior visual pathway is retinotopically consistent with the visual field defects and retinal nerve fiber layer loss in glaucoma. Examples of this anterograde transsynaptic degeneration has been demonstrated in several clinical, pathologic, and 
radiologic studies. In a clinicopathologic case study of a 79year-old man with POAG, the clinical manifestations of glaucoma, which included his inferior neuroretinal rim atrophy in the optic disc and his superior visual field defect, correlated with the post-mortem pathological findings of neuronal atrophy in the intracranial optic nerves, the posterior lateral part of the LGN (Figure 2), and the primary visual cortex below the calcarine sulcus. ${ }^{53}$ Furthermore, magnetic resonance imaging (MRI) evidence of LGN atrophy was shown to correlate with neuropathologic findings showing neuronal shrinkage and loss associated with reactive astrogliosis in the LGN of experimental primate glaucoma models. ${ }^{50,54,55}$ Deafferentation of the larger nerve fibers in this glaucoma model was followed by atrophy in the relay neurons and their dendrites in both the magnocellular and parvocellular layers of the LGN ${ }^{56,57}$ In another study of 18 patients with POAG compared with 18 age- and sex-matched healthy controls, ${ }^{58}$ ganglion cell-inner plexiform layer thinning significantly correlated with contralateral LGN volume reduction on 7-Tesla MRI.

Further evidence for a transsynaptic degenerative process has been shown by the axonal degeneration of the visual pathway from the LGN to the primary visual cortex on neuroimaging studies. ${ }^{57}$ Compared to normal controls, significant atrophy of the optic tracts, optic radiations, ${ }^{58}$ and the occipital white matter ${ }^{59,60}$ in POAG patients was seen on 3-Tesla diffusion tensor imaging. The degree of

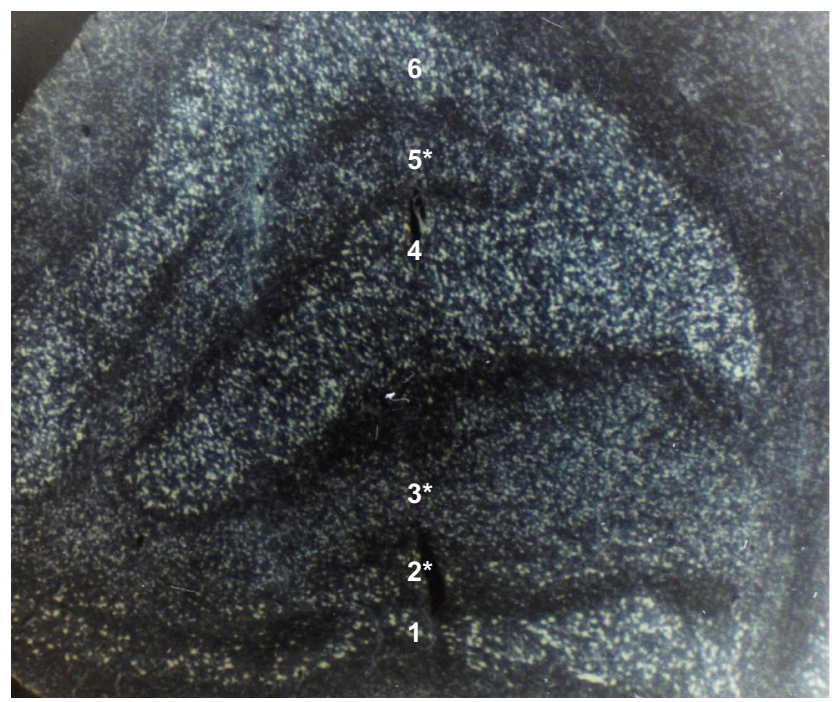

Figure 2 Coronal section of the left lateral geniculate nucleus in a 72-year-old patient after 8 years of optic atrophy from absolute glaucoma in the left eye. The smaller cells with less staining in ipsilateral layers 2, 3, and 5 (marked with an asterisk) represent transsynaptic atrophy. Layers $I$ and 2 are the magnocellular layers. Layers 3 to 6 are the parvocellular layers. (Contrast-enhanced grayscale photo of a Nissl-stained specimen at 20x magnification; unpublished observation). visual cortical thinning correlated with the severity of RNFL thinning. ${ }^{61}$ In a more recent study using pixelbased analysis of the white matter visual pathway in 12 patients with POAG compared with 16 healthy controls, the optic tracts had significantly reduced fiber density (representing early microstructural axonal changes) and fiber-bundle cross-sectional area (representing later macrostructural axonal alterations) in the glaucomatous patients compared with controls. ${ }^{62}$ The more advanced white matter degeneration in the optic tracts of glaucomatous patients compared with that in the optic radiations implies that transsynaptic degeneration might account for the anterior to posterior visual pathway progression of glaucoma. Furthermore, functional MRI studies have also shown decreased activity in the primary visual cortex using blood-oxygen-level-dependent (BOLD) contrast. ${ }^{63,64}$

In addition to the transsynaptic degenerative changes in the brain and eye, other brain regions beyond the visual cortex have been shown to have reduced volumes. In 18 POAG patients, the left temporal and right nasal RNFL were found to be significantly thinner than the right temporal and left nasal RNFL. ${ }^{65}$ The voxel-based morphometry and diffusion tensor imaging in these patients showed significant volume reduction in the left visual cortex, the left LGN, and the intracranial portion of the optic nerves and chiasma. Fractional anisotropy was significantly decreased in the inferior frontal-occipital fasciculus, the longitudinal and inferior frontal fasciculi, the putamen, the caudate nucleus, the anterior and posterior thalamic radiations, and the anterior and posterior limbs of the internal capsule of the left hemisphere $(\mathrm{P}<0.05){ }^{65}$ In 57 patients with POAG, ranging from the earliest stages to the end stages (absolute glaucoma), compared with 29 agematched normal controls, the disruption of anatomical connectivity along the visual pathway and in the nonvisual white matter tracts was significantly correlated with the stages of POAG based on RNFL thinning and visual field defects. ${ }^{66}$ Functional connectivity was decreased in the visual and working memory networks, but it was increased in the default mode and subcortical networks. Both the anatomical connectivity and the functional connectivity were already affected in the early stage of POAG. Nonvisual regions of the brain, such as the hippocampus and the frontal cortex, also progressed with advancing POAG stage. ${ }^{66}$ These observations of concomitant nonvisual pathway abnormalities in POAG patients could reflect pathology from comorbid conditions, such as AD or other types of dementias. Furthermore, the defective 
metabolism of APP secondary to the APOE gene mutation in POAG might account for the cognitive dysfunction that was shown to be significantly associated with POAG in African-Americans. ${ }^{67}$ Whether transsynaptic neurodegeneration in human glaucoma occurs via the focal adhesion pathway and/or the transsynaptic spreading of tau will require further detailed temporal and spatial transcriptomics and proteomic studies to decipher the regulatory pathways controlling beta-amyloid and tau metabolism in the retina and in the brain.

\section{Conclusion}

Lowering IOP remains to be the most modifiable risk factor for the successful treatment of POAG. However, future treatments will be developed to address the nonIOP-related factors of POAG to delay disease progression in the eye and in the brain. Structural and functional changes, such as inner retinal layer thinning, disc cupping, and reduced choline levels in the visual cortex from glaucomatous degeneration have been demonstrated to be already present in the brain before detectable visual field defects. $^{68}$ The underlying genetic and metabolic pathways of transsynaptic neurodegeneration in glaucoma remains to be elucidated. If tauopathy is considered as a final common pathway for neurodegenerative diseases, then therapies should be aimed at these downstream pathways, in addition to the preventive therapies aimed at upstream ones. Future investigations are needed to search for more comprehensive therapeutic strategies that address not only the elevated IOP risk factors, but also the non-IOP-related genetic regulatory pathways contributing to glaucomatous neurodegeneration in the eye and in the brain. Currently, GAL-101, a novel small molecule that prevents and reverses the synaptotoxic effects of $A B$ $1-42$, is being investigated as a promising therapy for glaucoma. ${ }^{69}$

\section{Disclosure}

The authors report no conflicts of interest in this work.

\section{References}

1. Almasieh M, Wilson AM, Morquette B, Cueva Vargas JL, Di Polo A. The molecular basis of retinal ganglion cell death in glaucoma. Prog Retin Eye Res. 2012;31(2):152-181. doi:10.1016/j.preteyeres.2011.11. 002

2. Dourlen P, Kilinc D, Malmanche N, Chapuis J, Lambert JC. The new genetic landscape of Alzheimer's disease: from amyloid cascade to genetically driven synaptic failure hypothesis? Acta Neuropathol. 2019;138(2):221-236. doi:10.1007/s00401-019-02004-0
3. McKee AC, Cantu RC, Nowinski CJ, et al. Chronic traumatic encephalopathy in athletes: progressive tauopathy after repetitive head injury. J Neuropathol Exp Neurol. 2009;68(7):709-735. doi:10.1097/NEN.0b0 13e3181a9d503

4. Hauser MA, Allingham RR, Aung T, Genetics of Glaucoma in People of African Descent C. Association of genetic variants with primary open-angle glaucoma among individuals with African ancestry. JAMA. 2019;322(17):1682-1691. doi:10.1001/jama.2019. 16161

5. Wang Y, Zhou YF, Zhao BY, Gu ZY, Li SL. Apolipoprotein E gene epsilon4epsilon4 is associated with elevated risk of primary open angle glaucoma in Asians: a meta-analysis. BMC Med Genet. 2014;15(1):60. doi:10.1186/1471-2350-15-60

6. Gatz M, Reynolds CA, Fratiglioni L, et al. Role of genes and environments for explaining Alzheimer disease. Arch Gen Psychiatry. 2006;63(2):168-174. doi:10.1001/archpsyc.63.2.168

7. Xu YX, Wang HQ, Yan J, Sun XB, Guo JC, Zhu CQ. Antibody binding to cell surface amyloid precursor protein induces neuronal injury by deregulating the phosphorylation of focal adhesion signaling related proteins. Neurosci Lett. 2009;465(3):276-281. doi:10.10 16/j.neulet.2009.09.022

8. Uhasz GJ, Barkoczi B, Vass G, et al. Fibrillar Abeta (1-42) enhances NMDA receptor sensitivity via the integrin signaling pathway. J Alzheimers Dis. 2010;19(3):1055-1067. doi:10.3233/JAD-20101301

9. Woo JA, Jung AR, Lakshmana MK, et al. Pivotal role of the RanBP9-cofilin pathway in Abeta-induced apoptosis and neurodegeneration. Cell Death Differ. 2012;19(9):1413-1423. doi:10.10 38/cdd.2012.14

10. Woo JA, Roh SE, Lakshmana MK, Kang DE. Pivotal role of RanBP9 in integrin-dependent focal adhesion signaling and assembly. FASEB J. 2012;26(4):1672-1681. doi:10.1096/fj.11-194423

11. Woo JA, Zhao X, Khan H, et al. Slingshot-cofilin activation mediates mitochondrial and synaptic dysfunction via Abeta ligation to beta1-integrin conformers. Cell Death Differ. 2015;22(6):10 69-1070. doi:10.1038/cdd.2015.41

12. Yuan $\mathrm{P}$, Condello $\mathrm{C}$, Keene $\mathrm{CD}$, et al. TREM2 haplodeficiency in mice and humans impairs the microglia barrier function leading to decreased amyloid compaction and severe axonal dystrophy. Neuron. 2016;92(1):252-264. doi:10.1016/j.neuron.2016.09.016

13. Neve RL, Harris P, Kosik KS, Kurnit DM, Donlon TA. Identification of cDNA clones for the human microtubule-associated protein tau and chromosomal localization of the genes for tau and microtubule-associated protein 2. Brain Res. 1986;387(3):271-280. doi:10.1016/0169-328x(86)90033-1

14. Andreadis A. Tau gene alternative splicing: expression patterns, regulation and modulation of function in normal brain and neurodegenerative diseases. Biochim Biophys Acta. 2005;1739(2-3):91-103. doi:10.1016/j.bbadis.2004.08.010

15. Caillet-Boudin ML, Buee L, Sergeant N, Lefebvre B. Regulation of human MAPT gene expression. Mol Neurodegener. 2015;10(1):28. doi:10.1186/s13024-015-0025-8

16. Caceres A, Kosik KS. Inhibition of neurite polarity by tau antisense oligonucleotides in primary cerebellar neurons. Nature. 1990;343 (6257):461-463. doi:10.1038/343461a0

17. Cleveland DW, Hwo SY, Kirschner MW. Purification of tau, a microtubule-associated protein that induces assembly of microtubules from purified tubulin. J Mol Biol. 1977;116(2):207-225. doi:10.1016/ 0022-2836(77)90213-3

18. Lee S, Kim W, Li Z, Hall GF. Accumulation of vesicle-associated human tau in distal dendrites drives degeneration and tau secretion in an in situ cellular tauopathy model. Int $J$ Alzheimers Dis. 2012;2012:172837.

19. Congdon EE, Sigurdsson EM. Tau-targeting therapies for Alzheimer disease. Nat Rev Neurol. 2018;14(7):399-415. doi:10.1038/s41582-0180013-z 
20. Laws SM, Hone E, Gandy S, Martins RN. Expanding the association between the APOE gene and the risk of Alzheimer's disease: possible roles for APOE promoter polymorphisms and alterations in APOE transcription. J Neurochem. 2003;84(6):1215-1236. doi:10.1046/ j.1471-4159.2003.01615.x

21. Saadat M. Apolipoprotein E (APOE) polymorphisms and susceptibility to breast cancer: a meta-analysis. Cancer Res Treat. 2012;44 (2):121-126. doi:10.4143/crt.2012.44.2.121

22. Corder EH, Saunders AM, Strittmatter WJ, et al. Gene dose of apolipoprotein E type 4 allele and the risk of Alzheimer's disease in late onset families. Science. 1993;261(5123):921-923. doi:10.11 26/science. 8346443

23. Schmechel DE, Saunders AM, Strittmatter WJ, et al. Increased amyloid beta-peptide deposition in cerebral cortex as a consequence of apolipoprotein E genotype in late-onset Alzheimer disease. Proc Natl Acad Sci U S A. 1993;90(20):9649-9653. doi:10.1073/pnas.90.20. 9649

24. Margeta MA, Letcher SM, Igo RP Jr, et al. Association of APOE with primary open-angle glaucoma suggests a protective effect for APOE \&4. Invest Ophthalmol Vis Sci. 2020;61(8):3. doi:10.1167/ iovs.61.8.3

25. Chen M, Yu X, Xu J, et al. Association of gene polymorphisms with primary open angle glaucoma: a systematic review and meta-analysis. Invest Ophthalmol Vis Sci. 2019;60(4):1105-1121. doi:10.1167/iovs.18-25922

26. Bayer AU, Keller ON, Ferrari F, Maag KP. Association of glaucoma with neurodegenerative diseases with apoptotic cell death: alzheimer's disease and Parkinson's disease. Am J Ophthalmol. 2002;133 (1):135-137. doi:10.1016/S0002-9394(01)01196-5

27. Helmer C, Malet F, Rougier MB, et al. Is there a link between open-angle glaucoma and dementia? The three-city-alienor cohort Ann Neurol. 2013;74(2):171-179. doi:10.1002/ana.23926

28. Tamura H, Kawakami H, Kanamoto T, et al. High frequency of openangle glaucoma in Japanese patients with Alzheimer's disease. J Neurol Sci. 2006;246(1-2):79-83. doi:10.1016/j.jns.2006.02.009

29. Kirby E, Bandelow S, Hogervorst E. Visual impairment in Alzheimer's disease: a critical review. J Alzheimers Dis. 2010;21 (1):15-34. doi:10.3233/JAD-2010-080785

30. Bossy-Wetzel E, Schwarzenbacher R, Lipton SA. Molecular pathways to neurodegeneration. Nat Med. 2004;10(Suppl):S2-9. doi:10. $1038 / \mathrm{nm} 1067$

31. Zhu X, Zhou W, Cui Y, et al. Pilocarpine protects cobalt chloride-induced apoptosis of RGC-5 cells: involvement of muscarinic receptors and HIF-1 alpha pathway. Cell Mol Neurobiol. 2010;30 (3):427-435. doi:10.1007/s10571-009-9467-2

32. Gupta N, Fong J, Ang LC, Yucel YH. Retinal tau pathology in human glaucomas. Can J Ophthalmol. 2008;43(1):53-60. doi:10.3129/i07185

33. Yoneda S, Hara H, Hirata A, Fukushima M, Inomata $Y$, Tanihara $H$. Vitreous fluid levels of beta-amyloid((1-42)) and tau in patients with retinal diseases. Jpn J Ophthalmol. 2005;49(2):106-108. doi:10.10 07/s10384-004-0156-x

34. Yan Z, Liao H, Chen $\mathrm{H}$, et al. Elevated intraocular pressure induces amyloid-beta deposition and tauopathy in the lateral geniculate nucleus in a monkey model of glaucoma. Invest Ophthalmol Vis Sci. 2017;58(12):5434-5443. doi:10.1167/iovs.17-22312

35. Chiasseu M, Cueva Vargas JL, Destroismaisons L, Vande Velde C, Leclerc N, Di Polo A. Tau accumulation, altered phosphorylation, and missorting promote neurodegeneration in glaucoma. J Neurosci. 2016;36:5785-5798.

36. Leger F, Fernagut PO, Canron MH, et al. Protein aggregation in the aging retina. $J$ Neuropathol Exp Neurol. 2011;70(1):63-68. doi:10.1097/NEN.0b013e31820376cc

37. Ghoshal N, Garcia-Sierra F, Wuu J, et al. Tau conformational changes correspond to impairments of episodic memory in mild cognitive impairment and Alzheimer's disease. Exp Neurol. 2002;177:475-493.
38. Kuszczyk M, Gordon-Krajcer W, Lazarewicz JW. Homocysteineinduced acute excitotoxicity in cerebellar granule cells in vitro is accompanied by PP2A-mediated dephosphorylation of tau. Neurochem Int. 2009;55:174-180.

39. Lasagna-Reeves CA, Castillo-Carranza DL, Sengupta U, et al. Identification of oligomers at early stages of tau aggregation in Alzheimer's disease. FASEB J. 2012;26(5):1946-1959. doi:10.1096/ fj.11-199851

40. Patterson KR, Remmers C, Fu Y, et al. Characterization of prefibrillar tau oligomers in vitro and in alzheimer disease. J Biol Chem. 2011;286(26):23063-23076. doi:10.1074/jbc.M111.237974

41. Scholz T, Mandelkow E. Transport and diffusion of Tau protein in neurons. Cell Mol Life Sci. 2014;71(16):3139-3150. doi:10.1007/ s00018-014-1610-7

42. Nilsen LH, Rae C, Ittner LM, Gotz J, Sonnewald U. Glutamate metabolism is impaired in transgenic mice with tau hyperphosphorylation. J Cereb Blood Flow Metab. 2013;33 (5):684-691. doi:10.1038/jcbfm.2012.212

43. Agostinone J, Di Polo A. Retinal ganglion cell dendrite pathology and synapse loss: implications for glaucoma. Prog Brain Res. 2015;220:199-216.

44. Jakobs TC, Libby RT, Ben Y, John SW, Masland RH. Retinal ganglion cell degeneration is topological but not cell type specific in DBA/2J mice. J Cell Biol. 2005;171(2):313-325. doi:10.1083/jcb.200506099

45. Della Santina L, Inman DM, Lupien CB, Horner PJ, Wong RO. Differential progression of structural and functional alterations in distinct retinal ganglion cell types in a mouse model of glaucoma. J Neurosci. 2013;33(44):17444-17457. doi:10.1523/JNEUROSCI.5461-12.2013

46. Dandona L, Hendrickson A, Quigley HA. Selective effects of experimental glaucoma on axonal transport by retinal ganglion cells to the dorsal lateral geniculate nucleus. Invest Ophthalmol Vis Sci. 1991;32 (5):1593-1599.

47. Nickells RW, Howell GR, Soto I, John SW. Under pressure: cellular and molecular responses during glaucoma, a common neurodegeneration with axonopathy. Annu Rev Neurosci. 2012;35(1):153-179. doi:10.1146/annurev.neuro.051508.135728

48. Martin KR, Quigley HA, Valenta D, Kielczewski J, Pease ME. Optic nerve dynein motor protein distribution changes with intraocular pressure elevation in a rat model of glaucoma. Exp Eye Res. 2006;83:255-262.

49. Yucel YH, Zhang Q, Weinreb RN, Kaufman PL, Gupta N. Atrophy of relay neurons in magno- and parvocellular layers in the lateral geniculate nucleus in experimental glaucoma. Invest Ophthalmol Vis Sci. 2001;42(13):3216-3222.

50. Weber AJ, Chen H, Hubbard WC, Kaufman PL. Experimental glaucoma and cell size, density, and number in the primate lateral geniculate nucleus. Invest Ophthalmol Vis Sci. 2000;41:1370-1379.

51. Hell JW. CaMKII: claiming center stage in postsynaptic function and organization. Neuron. 2014;81(2):249-265. doi:10.1016/j.neuron. 2013.12.024

52. Yamagata Y, Kobayashi S, Umeda T, et al. Kinase-dead knock-in mouse reveals an essential role of kinase activity of $\mathrm{Ca} 2+/$ calmodulin-dependent protein kinase IIalpha in dendritic spine enlargement, long-term potentiation, and learning. $J$ Neurosci. 2009;29 (23):7607-7618. doi:10.1523/JNEUROSCI.0707-09.2009

53. Gupta N, Ang LC. Human glaucoma and neural degeneration in intracranial optic nerve, lateral geniculate nucleus, and visual cortex. $\mathrm{Br}$ J Ophthalmol. 2006;90(6):674-678. doi:10.1136/bjo.2005.086769

54. Yucel YH, Gupta N, Zhang Q, Mizisin AP, Kalichman MW, Weinreb RN. Memantine protects neurons from shrinkage in the lateral geniculate nucleus in experimental glaucoma. Arch Ophthalmol. 2006;124(2):217-225. doi:10.1001/archopht.124.2.217

55. Shimazawa M, Tomita G, Taniguchi T, et al. Morphometric evaluation of changes with time in optic disc structure and thickness of retinal nerve fibre layer in chronic ocular hypertensive monkeys. Exp Eye Res. 2006;82(3):427-440. doi:10.1016/j.exer.2005.08.001 
56. Ly T, Gupta N, Weinreb RN, Kaufman PL, Yucel YH. Dendrite plasticity in the lateral geniculate nucleus in primate glaucoma. Vision Res. 2011;51(2):243-250. doi:10.1016/j.visres.2010.08.003

57. Yucel YH, Zhang Q, Weinreb RN, Kaufman PL, Gupta N. Effects of retinal ganglion cell loss on magno-, parvo-, koniocellular pathways in the lateral geniculate nucleus and visual cortex in glaucoma. Prog Retin Eye Res. 2003;22(4):465-481. doi:10.1016/S1350-9462(03)00026-0

58. Chen Z, Lin F, Wang J, et al. Diffusion tensor magnetic resonance imaging reveals visual pathway damage that correlates with clinical severity in glaucoma. Clin Exp Ophthalmol. 2013;41(1):43-49. doi:10.1111/j.1442-9071.2012.02832.x

59. Lu P, Shi L, Du H, et al. Reduced white matter integrity in primary open-angle glaucoma: a DTI study using tract-based spatial statistics. J Neuroradiol. 2013;40(2):89-93. doi:10.1016/j.neurad.2012.04.001

60. Bogorodzki P, Piatkowska-Janko E, Szaflik J, Szaflik JP, Gacek M, Grieb P. Mapping cortical thickness of the patients with unilateral end-stage open angle glaucoma on planar cerebral cortex maps. PLoS One. 2014;9(4):e93682. doi:10.1371/journal.pone.0093682

61. Yu L, Xie B, Yin X, et al. Reduced cortical thickness in primary open-angle glaucoma and its relationship to the retinal nerve fiber layer thickness. PLoS One. 2013;8(9):e73208. doi:10.1371/journal. pone. 0073208

62. Haykal S, Curcic-Blake B, Jansonius NM, Cornelissen FW. Fixelbased analysis of visual pathway white matter in primary open-angle glaucoma. Invest Ophthalmol Vis Sci. 2019;60(12):3803-3812. doi:10.1167/iovs.19-27447

63. Duncan RO, Sample PA, Weinreb RN, Bowd C, Zangwill LM. Retinotopic organization of primary visual cortex in glaucoma: a method for comparing cortical function with damage to the optic disk. Invest Ophthalmol Vis Sci. 2007;48(2):733-744. doi:10.1167/iovs.06-0773
64. Qing G, Zhang S, Wang B, Wang N. Functional MRI signal changes in primary visual cortex corresponding to the central normal visual field of patients with primary open-angle glaucoma. Invest Ophthalmol Vis Sci. 2010;51(9):4627-4634. doi:10.1167/iovs.094834

65. Zikou AK, Kitsos G, Tzarouchi LC, Astrakas L, Alexiou GA, Argyropoulou MI. Voxel-based morphometry and diffusion tensor imaging of the optic pathway in primary open-angle glaucoma: a preliminary study. AJNR Am J Neuroradiol. 2012;33(1):128-134. doi:10.3174/ajnr.A2714

66. Frezzotti P, Giorgio A, Toto F, De Leucio A, De Stefano N. Early changes of brain connectivity in primary open angle glaucoma. Hum Brain Mapp. 2016;37:4581-4596.

67. McCoskey M, Addis V, Goodyear K, et al. Association between primary open-angle glaucoma and cognitive impairment as measured by the montreal cognitive assessment. Neurodegener Dis. 2018;18 (5-6):315-322. doi:10.1159/000496233

68. Murphy MC, Conner IP, Teng CY, et al. Retinal structures and visual cortex activity are impaired prior to clinical vision loss in glaucoma. Sci Rep. 2016;6(1):31464. doi:10.1038/srep31464

69. Rammes G, Parsons CG. The Abeta aggregation modulator MRZ-99030 prevents and even reverses synaptotoxic effects of Abeta1-42 on LTP even following serial dilution to a 500:1 stoichiometric excess of Abeta1-42, suggesting a beneficial prion-like seeding mechanism. Neuropharmacology. 2020;179:108267. doi:10.1016/ j.neuropharm.2020.108267
Eye and Brain

\section{Publish your work in this journal}

Eye and Brain is an international, peer-reviewed, open access journal focusing on clinical and experimental research in the field of neuroophthalmology. All aspects of patient care are addressed within the journal as well as basic research. Papers covering original research, basic science, clinical and epidemiological studies, reviews and

Submit your manuscript here: https://www.dovepress.com/eye-and-brain-journal evaluations, guidelines, expert opinion and commentary, case reports and extended reports are welcome. The manuscript management system is completely online and includes a very quick and fair peerreview system, which is all easy to use. Visit http://www.dovepress. com/testimonials.php to read real quotes from published authors. 